Pacific Journal of Mathematics

NOTES ON GENERALIZED BOUNDARY VALUE PROBLEMS IN
BANACH SPACES. I. ADJOINT AND EXTENSION THEORY 


\title{
NOTES ON GENERALIZED BOUNDARY VALUE PROBLEMS IN BANACH SPACES, I ADJOINT AND EXTENSION THEORY
}

\author{
R. C. BROWN
}

Let $A: X \rightarrow Y$ be a densely defined closed operator where $X$ and $Y$ are Banach spaces. Let $F$ be a locally convex topological vector space and $H: X \rightarrow F$ an operator such that $N(H)$ and $D(A)$ have nontrivial intersection and $D\left(H^{*}\right)$ is total over $F$. We compute $A_{H}^{*}$ and $A_{H}^{*}$ where $A_{H}$ is the operator determined by $A$ on $N(H)$ and $A_{H}(x)=$ $(A x, H x)^{t}$.

We also characterize certain closed extensions of $A_{H}$ and the adjoints of these extensions. In particular application is made to the problem of determining self-adjoint extensions of symmetric operators restricted by boundary conditions in a Hilbert space.

1. Introduction. Suppose $X, Y$ and $A$ are as above. Let $H$ be an operator having domain in $X$ and range in a locally convex topological vector space (l.c.t.v.s.) $F$. Assume that $D(A) \cap N(H)$ is nontrivial. Then the system

$$
\begin{aligned}
& A x=f \\
& H x=r
\end{aligned}
$$

is called a generalized boundary value problem (b.v.p). We call the first equation of (1.1) the operator part of the b.v.p. and the second the boundary condition. $H$ is the boundary operator. If $r=0$ the problem is said to be homogeneous, otherwise it is nonhomogeneous. In the nonhomogeneous case, (1.1) determines an operator $\mathscr{A}_{H}: X \rightarrow$ $Y \times F$ and in the homogeneous case an operator $A_{H} \subset A: X \rightarrow Y$ on

$$
D\left(A_{H}\right):=\{x \in D(A): H x=0\} .
$$

In this paper we are going to construct the adjoints $A_{I I}^{*}$ and $\mathscr{A}_{H}^{*}$ and compare their structure. Knowledge of $A_{H}^{*}$ and $\mathscr{A}_{H}^{*}$ yield at once statements of Fredholm alternative solvability conditions for the original b.v.p. We will also be interested in the following extension problem. Suppose $A$ and $B: Y^{*} \rightarrow X^{*}$ are 1-1 and $B^{*} \supset A$. Let $K: Y^{*} \rightarrow G$ ( $G$ a l.c.t.v.s) be a boundary operator. Then (roughly speaking)

$$
A_{H} \subset A \subset B_{K}^{*}
$$

One can now ask for the structure of all closed extensions of $A_{H}$ 
which are restrictions of $B_{K}^{*}$. In the special case when $X=Y=$ Hilbert space and $H=K A_{H}$ is symmetric, and the problem amounts the determination of all self-adjoint extensions of $A_{H}$.

Both the adjoint and extension problems for generalized b.v.p. have been investigated in several recent papers, notably [5], [6], [9]. In [6] for example $A$ is a linear relation in $X \times Y$ and $A_{H}=A \cap{ }^{*} B$ where ${ }^{*} B$ is the preadjoint of a finite dimensional subspace $B$ in $Y^{*} \times X^{*}$. Such a representation is always possible if $H$ is continuous on $G(A)$ and $F$ is finite dimensional. This "subspace" interpretation of $A_{H}$ leads to an elegant construction of $\left(A \cap \cap^{*} B\right)^{*}$ and also to a solution of the extension problem when (in our notation)

$$
\operatorname{dim} G\left(A_{K}^{*}\right) / G\left(A_{H}\right)<\infty .
$$

The contributions of the present paper are twofold. In the first place we extend the theory by letting $F$ be an infinite dimensional t.v.s. Secondly there is a change in point of view distinguishes this paper from [6]. We represent the boundary condition directly in terms of the null space of the boundary operator given in the problem. Thus we bypass the task of finding ${ }^{*} B$. Furthermore because much of the theory presented here is an abstraction of ideas in the writers earlier papers [2], [3] on Stieltjes b.v.p., our technique gives simple formulas and characterizations which are easy to apply both to this and other types of concrete b.v.p.

We now briefly summarize the paper. Notational conventions and fundamental definitions are introduced in $\S 2$. Here in particular we discuss the notion of an abstract boundary condition and prove that every closed restriction of a closed linear relation $A$ is an " $A_{H}$ " with reference to a certain l.c.t.v.s. $F$ and a boundary operator $H$. Section 3 is devoted to the computation of $A_{H}^{*} . \quad F$ is assumed to be both finite and infinite dimensional; and significant differences in the structure of the adjoint are pointed out. In the infinite dimensional case we first assume that $G\left(-A^{*}\right)$ is complemented (Theorem 3.6). However since this is an inconvenient hypothesis in a non Hilbert space setting we investigate several ways in which it can be weakened.

The final result (Corollary 3.14 ) is an especially simple construction of $A_{H}^{*}$ when $A$ is $1-1$. We illustrate this construction by an example. Section 4 solves the extension problem mentioned above: first in the finite dimensional case and secondly for extensions having closed range. Finally $\S 5$ treats the nonhomogeneous case. $\mathscr{A}_{H}^{*}$ is determined and its structure compared with $A_{H}^{*}$.

Although we occasionally illustrate the theory with examples, most applications to Stieltjes, and interface b.v.p, to evolution and 
functional differential operators, and to calculus of variations and control theory (extending some preliminary ideas already presented in [3]) will be reserved for a forthcoming paper.

2. Notation and preliminaries. If $T$ is a linear operator or relation $D(T), R(T), N(T)$ will stand for its domain, range, and null space respectively. $T^{*}$ denotes the congugate transpose, dual, adjoint or preadjoint of a matrix, space, or linear mapping according to the context, (we write the transpose of a matrix $M$ as $M^{t}$ ). The notations $\bar{S}$ or the terms "closed", "continuous" signify weak* closure of a set $S \subset X$ or weak* continuity if $X$ is a dual space; otherwise we are referring to the closure of $S$ or strong continuity in the topo$\log y$ of $X$. Similarly $S^{\perp}$ means either the preannihilator, i.e.,

$$
\left\{s \in X:\left[s, s^{\prime}\right]=0, s^{\prime} \in S\right\}
$$

or the annihilator of $S$, i.e.,

$$
\left\{s \in X^{*}:\left[s^{\prime}, s\right]=0, s^{\prime} \in S\right\} .
$$

If $X$ is a space and $X^{*}$ is its dual $[\cdot, \cdot]$ signifies the sesquilinear pairing on $X \times X^{*}$ given by

$$
\left[x, x^{*}\right] \overline{=x^{*}(x)} \text {. }
$$

If $X, Y$ are spaces and $X^{+}$is total on $X$ and $Y^{+}$is total on $Y$ we define a pairing on $(X \times Y) \times\left(X^{+} \times Y^{+}\right)$by

$$
\left[(x, y),\left(x^{+}, y^{+}\right)\right]:=\left[y, y^{+}\right]+\left[x, x^{+}\right] \text {. }
$$

If $X$ and $Y$ are normed we define a norm as $X \times Y$ by

$$
\|(x, y)\|:=\|x\|+\|y\| \text {. }
$$

A linear relation $A: X \rightarrow Y$ where $X, Y$ are linear spaces is a set valued mapping whose graph $G(A)$ is a subspace of $X \times Y$. Unless otherwise mentioned all relations are assumed closed; i.e., to have closed graph. For $\alpha \in D(A)$ we denote the image of $\alpha$ in $R(A)$ by $A(\alpha)$; the notation $\left(\alpha, A_{\alpha}\right)$ will signify an arbitrary element in $G(A)$ such that $A_{\alpha} \in A(\alpha)$. It is easily checked that $A(0)$ is a subspace of $R(A)$ and elements $\beta, \alpha \in A(\alpha)$ if and only if $\beta \equiv \alpha \bmod$ $A(0)$; i.e., the induced mapping $A^{\prime}: X \rightarrow X / A(0)$ is an operator. A relation is an operator if and only if it is single valued; i.e., if and only if $A(0)=0$. If $A$ is a closed operator $D(A)$ is a Banach space with respect to the graph topology defined by the norm

$$
\|x\|:=\|x\|_{X}+\|A x\|_{Y} .
$$

$A$ is then a continuous operator with respect to the graph topology. 
We will also write $B \subset A$ if $G(B) \subset G(A)$; in this case. $B$ is said to be a restriction of $A$ and $A$ is called an extension of $B$.

Definition 2.1. The adjoint $A^{*}: Y^{*} \rightarrow X^{*}$ of $A: X \rightarrow Y$ is the relation with graph

$$
\{(\alpha, \beta):[y, \alpha]-[x, \beta]=0 ;(x, y) \in A\} .
$$

Definition 2.2. The preadjoint of $B^{*}: Y^{*} \rightarrow X^{*}$ is the relation with graph

$$
\{(\alpha, \beta):[\alpha, y]-[\beta, x]=0 ;(x, y) \in B\} .
$$

As indicated above $A^{*}$ means either the adjoint or preadjoint of $A$ depending on the context. A complete discussion of the properties of adjoint and preadjoint relations may be found in [1] or [6]. We specifically mention here only a generalization for relations of the classical Banach closed range theorem for operators (see [6] for the proof).

TheOREM 2.3. If $A: X \rightarrow Y$ is a closed relation then norm closure of $R(A)$ is equivalent to both the norm and weak* closure of $R\left(A^{*}\right)$. Similarly if $B: Y^{*} \rightarrow X^{*}$ is a weak* closed relation the norm closure of $R\left(B^{*}\right)$ is equivalent to both the norm and weak* closure of $R(B)$.

Suppose $B$ is a (closed) restriction of a relation $A: X \rightarrow Y$. Define an operator

$$
H: G(A) \longrightarrow G(B)^{\perp *}
$$

by

$$
H(y, A y) \circ\langle\alpha, \beta\rangle=[A y, \alpha]+[y, \beta],(\alpha, \beta) \in G(B)^{\perp} .
$$

$G(B)^{\perp *}$ under the weak* topology is a l.c.t.v.s. By the definition of this topology $H$ is continuous. It is clear that the nullspace of $H$ is exactly $G(B)$. We fix these ideas with a definition.

DeFinition 2.4. Let $A: X \rightarrow Y$ be a relation and $F$ a l.c.t.v.s. Then an operator $H: X \times Y \rightarrow F$ such that $D(H) \supset G(A)$ is called a boundary operator provided $D\left(H^{*}\right)$ is total over $F$, and the condition $H(y, A y)=0$ is called a boundary condition.

In terms of Definition 2.4 the previous discussion has shown.

LemMA 2.5. $B$ is a closed restriction of $A$ if and only if 
$B=A_{H}$. The boundary operator $H$ is continuous with range in a l.c.t.v.s. If $A$ is an operator $H$ can be viewed as an operator such that $D(H) \supset D(A)$ which is continuous in the graph topology on $G(A)$.

The importance of Lemma 2.5 is "existential": every restriction of $A$ is determined by a certain "canonical" boundary condition. In most cases however a boundary operator $H$ is given a priori; it and the canonical operator supplied by the lemma may not be the same (only equivalent in the sense that their nullspaces are the same.) Indeed the canonical operator may be hard to find. Therefore the results in this paper will be expressed soley in terms of an arbitrary boundary operator considered to be given in the problem and Lemma 2.5 will be used only as a theorem proving tool.

We close this section by mentioning a simple result frequently used in the proofs of this paper.

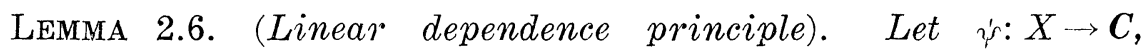
$i=1, \cdots, n$, and $\rho: X \rightarrow C$ be linear functionals such that

$$
N(\dot{\phi}) \supset \cap N\left(\dot{\psi}_{i}\right) \text {. }
$$

Then (provided $\phi \not \equiv 0)$

$$
\dot{\rho}=\sum c_{i} \psi_{i}
$$

where not all of the constants $c_{i}$ are zero.

Proof. See [10] p. 62 .

3. The adjoint of $A_{I I}$. Let $A: X \rightarrow Y$ be a closed densely defined operator and let $H$ be a boundary operator for $A$. In this section we determine $A_{H}^{*}$ in terms of $A^{*}$ and $H^{*}$.

As stated in Definition $2.4 X \times Y \supset D(H) \supset G(A)$. Hence $H^{*}: F^{*} \rightarrow$ $X^{*} \times Y^{*}$ is in general a relation (unless $H$ is densely defined) and $H^{*}(0)$ is a subspace of

$$
G\left(-A^{*}\right):=\left\{\left(y,-A^{*} y\right)\right\} .
$$

We have assumed that $D\left(H^{*}\right)$ is at least total over $F$. To see the significance of this assumption, let $\left(V_{\phi}, U_{\dot{\phi}}\right)$ denote an arbitrary representative in $H^{*}(\phi)$. Then

$$
\begin{aligned}
{[H(y, A y), \dot{\phi}] } & =\left[(A y, y), H^{*}(\dot{\phi})\right] \\
& =\left[A y, V_{\phi}\right]+\left[y, U_{\phi}\right] .
\end{aligned}
$$

Since $F$ endowed with the weak topology relative to $D\left(H^{*}\right)$ is a l.c.t.v.s (see [10] p. 62) such that $F^{*}=D\left(H^{*}\right)$ the above equation 
shows that $H: G(A) \rightarrow F$ is now "weakly" continuous. Thus (provided $D\left(H^{*}\right)$ is total) we can assume with no loss of generality that $H$ is continuous on $G(A)$ by redefining the topology on $F$ if neccessary.

DEFINITION 3.1.

$$
\begin{aligned}
& D_{H}^{+}:=D\left(A^{*}\right)+\left\{V_{\phi}: \phi \in F^{*}\right\} \\
& \left.\widetilde{D}_{I I}^{+}:=D\left(A^{*}\right)+\pi_{1} \overline{\left(R\left(H^{*}\right)\right.}\right)
\end{aligned}
$$

where $\pi_{1}$ denotes projection on $Y^{*}$.

$$
\begin{aligned}
& \dot{\phi}(z):=\left\{\dot{\phi}: z-V_{\dot{\phi}} \in D\left(A^{*}\right)\right\} \\
& \dot{\psi}(z):=\left\{\left(\psi_{1}, \psi_{2}\right) \in \overline{R\left(H^{*}\right)}: z-\psi_{1} \in D\left(A^{*}\right)\right\} .
\end{aligned}
$$

Further let $A_{I I}^{+}, \widetilde{A}_{I I}^{+}$be the relations in $Y^{*} \times X^{*}$ such that

$$
\begin{aligned}
& G\left(A_{H}^{+}\right):=\left\{\left(z, A^{*}\left(z-V_{\phi}\right)-U_{\dot{\phi}}\right): \dot{\phi} \in \dot{\phi}(z)\right\} \\
& G\left(\widetilde{A}_{I I}^{+}\right):=\left\{z, A^{*}\left(z-\psi_{1}\right)-\psi_{2}:\left(\psi_{1}, \dot{\psi}_{2}\right) \in \psi^{\prime}(z)\right\} .
\end{aligned}
$$

LEMMA 3.2. The following is true:

(1) $A_{I I}^{+}$is well defined modulo representatives $\left(V_{\phi}, U_{\phi}\right)$ in $H^{*}(\dot{\phi})$.

(2) $\widetilde{D}_{H}^{+} \subset D\left(A^{*}\right)+\left\{\overline{V_{\dot{\phi}}: \phi \in F^{*}}\right\}$.

(3) $A^{*} \subset A_{I I}^{+} \subset \widetilde{A}_{I I}^{+}$.

(4) $A_{I I}^{+}(0)=\left\{A^{*}\left(V_{\dot{\varphi}}\right)-U_{\dot{\varphi}}: \phi \in \dot{\phi}(0)\right\}$.

$\widetilde{A}_{\text {II }}^{+}(0)=\overline{A_{I I}^{+}(0)}$

$$
=\left\{A^{*}\left(\psi_{1}\right)-\psi_{2}:\left(\psi_{1}, \psi_{2}\right) \in \psi^{\prime}(0)\right\} .
$$

Proof. We demonstrate only (1) since (2)-(4) are immediate from the definition. Suppose $\left(V_{\phi}, U_{\phi}\right),\left(V_{\phi}^{\prime}, U_{\phi}^{\prime}\right) \in H^{*}(\phi)$. Since

$$
\left(V_{\phi}-V_{\dot{\phi}}^{\prime}, U_{\dot{\phi}}-U_{\dot{\phi}}^{\prime}\right) \in H^{*}(0)=G\left(-A^{*}\right),
$$

it is clear that

$$
U_{\dot{\phi}}^{\prime}-U_{\dot{\phi}}^{\prime}=A^{*}\left(V_{\dot{\phi}}-V_{\dot{\varphi}}^{\prime}\right)
$$

and

$$
\begin{gathered}
A^{*}\left(z-V_{\phi}^{\prime}\right)-U_{\phi}^{\prime}-\left(A^{*}\left(z-V_{\phi}\right)-U_{\phi}\right) \\
=A^{*}\left(V_{\phi}-V_{\phi}^{\prime}\right)-\left(U_{\phi}-U_{\phi}^{\prime}\right)=0 .
\end{gathered}
$$

THEOREM 3.3. $A_{I I}^{+*}=A_{I I}$.

Proof. Since $A_{I I}^{+} \supset A^{*}, A_{I I}^{+*} \subset A^{* *}=A$. Thus if $(y, A y) \in G\left(A_{H}^{+}\right)$

$$
[A y, z]-\left[y, A_{I I}^{+} z\right]=[A y, z]-\left[y, A^{*}\left(z-V_{\phi}\right)-U_{\dot{\phi}}\right]
$$




$$
\begin{aligned}
& =\left[A y, V_{\phi}\right]+\left[y, U_{\phi}\right] \\
& =[H y, \phi] \\
& =0
\end{aligned}
$$

Since $D\left(H^{*}\right)$ is total, $H y=0$ and $y \in D\left(A_{H}\right)$. Thus $A_{H}^{+*} \subset A_{H}$. However if $y \in D\left(A_{H}\right)$ (so that $H y=0$ ), the above computation shows immediately that $A_{H} \subset A_{H}^{+*}$.

Theorem 3.4. If $F$ is finite dimensional $A_{H}^{+}=A_{H}^{*}$.

Proof. In view of Theorem 3.3 it is only necessary to prove that $G\left(A_{H}^{*}\right) \subset A_{I I}^{+}$. Let $(\alpha, \beta) \in G\left(A_{H}^{*}\right)$. Define the functional $\psi_{\alpha \beta}$ : $G\left(A^{-1}\right) \rightarrow C$ by

$$
\psi_{\alpha \beta}(x):=[A x, \alpha]-[x, \beta]
$$

Since

$$
N\left(\psi_{\alpha \beta}\right) \supset G\left(A_{H}^{-1}\right)=N(H) \cap G\left(A^{-1}\right),
$$

it follows by Lemma 2.6 that

$$
\begin{aligned}
{[A x, \alpha]-[x, \beta] } & =[H(A x, x), \phi] \\
& =\left[(A x, x), H^{+}(\phi)\right] \\
& =\left[A x, V_{\phi}\right]+\left[x, U_{\phi}\right]
\end{aligned}
$$

for some $\phi$ in $F^{*}$. Transposing we conclude that $\alpha-V_{\phi} \in D\left(A^{*}\right)$ and $\beta=A^{*}\left(\alpha-V_{\phi}\right)-U_{\phi}$.

We now consider the case where $F$ is infinite dimensional.

LEMMA 3.5. If $G\left(-A^{*}\right)$ is complemented there exists an operator $H^{+}: F^{*} \rightarrow G\left(-A^{*}\right)^{\prime}$ such that

$$
[H y, \phi]=\left[(A y, y), H_{\phi}^{+}\right] .
$$

Proof. Let

$$
H^{+}(\phi):=(I-P) H^{*}(\phi)
$$

where $P$ is the weak* continuous projection of $Y^{*} \times X^{*}$ onto $G\left(-A^{*}\right)$. That elements of $H_{\phi}^{+}$satisfy (3.1) is obvious. If $\left(V_{\phi}^{+}, U_{\phi}^{+}\right),\left(V_{\phi}^{+\prime}, U_{\phi}^{+\prime}\right) \in$ $H^{+}(\phi)$ then it follows from (3.1) that

$$
\left(V_{\phi}^{+}-V_{\phi}^{+}, U_{\phi}^{+\prime}-U_{\phi}^{+\prime}\right) \in G\left(-A^{*}\right) .
$$

Since

$$
\left(V_{\phi}^{+}-V_{\phi}^{+\prime}, U_{\phi}^{+\prime}-U_{\phi}^{+\prime}\right) \in G\left(-A^{*}\right)
$$


by (3.2),

$$
V_{\phi}^{+}=V_{\phi}^{+\prime}=U_{\phi}^{+}=U_{\phi}^{+\prime}
$$

THEOREM 3.6. If $G\left(-A^{*}\right)$ is complemented $A_{H}^{*}=\widetilde{A}_{H}^{+}$.

Proof. By Theorem 3.3 and the standard theory of adjoints $A_{H}^{*}=\overline{A_{H}^{+}}$. Thus it suffices to show that $\overline{A_{H}^{+}}=\widetilde{A}_{H}^{+}$. To this end suppose that $\left\langle z_{n}\right\rangle$ is a net in $D_{H}^{+}$converging to $z$ in the weak topology of $Y^{*}$ and that $\beta_{n} \in A_{H}^{+} z_{n}$ is the general term of a net converging to $\beta$ in the weak* topology of $X^{*}$. Using Lemma 3.5 we write

$$
\left(V_{\phi}, U_{\phi}\right)=\left(V_{\phi}^{+}, U_{\phi}^{+}\right)+\left(\widetilde{V}_{\phi}, U_{\phi}\right)
$$

where

$$
\begin{aligned}
& \left(V_{\phi}^{+}, U_{\phi}^{+}\right)=(1-P)\left(V_{\phi}, U_{\phi}\right) \in G\left(-A^{*}\right)^{\prime} \\
& \left(\widetilde{V}_{\phi}, \widetilde{U}_{\phi}\right)=P\left(V_{\phi}, U_{\phi}\right) \in G\left(-A^{*}\right)
\end{aligned}
$$

Since

$$
z_{n}-V_{\phi_{n}}^{+} \in D\left(A^{*}\right)
$$

and

$$
\left.\beta_{n} \in A^{*}\left(z_{n}-V_{\phi_{n}}\right)-U_{\phi_{n}}=A^{*}\left(z_{n}-V_{\phi_{n}}^{+}\right)-U_{\phi_{n}}^{+}\right),
$$

(c.f. Lemma 3.2(1)),

$$
\left(z_{n}-V_{\phi_{n}}^{+},-\left(\beta_{n}+U_{\phi_{n}}^{+}\right)\right) \in G\left(-A^{*}\right) .
$$

Adding (3.3) and (3.4) we obtain $\left(z_{n},-\beta_{n}\right)$. Hence

$$
\begin{aligned}
& \left(V_{\phi_{n}}^{+}, U_{\phi_{n}}^{+}\right)=(I-P)\left(z_{n},-\beta_{n}\right) \\
& \left(z_{n}-V_{\phi_{n}}^{+},-\left(B_{n}+U_{\phi_{n}}^{+}\right)\right)=P\left(z_{n}, \beta_{n}\right) .
\end{aligned}
$$

We conclude that the net $\left\langle V_{\phi_{n}}^{+}, U_{\phi_{n}}^{+}\right\rangle$converges weak ${ }^{*}$ to $\left(\psi_{1}, \psi_{2}\right)$ in $\overline{R\left(H^{+}\right)} \subset \overline{R\left(H^{*}\right)}$. Finally (3.5), (3.6) and the closure of $G\left(-A^{*}\right)$ imply that

$$
\left(z-\psi_{1},-\left(\beta+\psi_{2}\right)\right) \in G\left(-A^{*}\right)
$$

i.e.,

$$
(z, \beta) \in G\left(\widetilde{A}_{H}^{+}\right)
$$

and thus $\overline{A_{H}^{+}} \subset \widetilde{A}_{H}^{+}$.

To show the reverse inclusion, suppose $(y, A y) \in G\left(A_{H}\right)$ and $\left(z, A^{*}\left(z-\psi_{1}\right)-\psi_{2}\right) \in G\left(A_{H}^{+}\right)$. Since 


$$
\left[A y, z-\psi_{1}\right]=\left[y, A^{*}\left(z-\psi_{1}\right)\right]
$$

it follows that

$$
[A y, z]-\left[y, A^{*}\left(z-\psi_{1}\right)-\psi_{2}\right]=\left[A y, \psi_{1}\right]+\left[y, \psi_{2}\right] .
$$

Since $\left(\psi_{1}, \psi_{2}\right) \in \overline{R\left(H^{*}\right)}$ and weak ${ }^{*}$ closed sets are also strongly closed, given $\varepsilon>0$ there exists $\left(V_{\phi_{\varepsilon}}, U_{\phi_{\varepsilon}}\right)$ such that

$$
\begin{aligned}
& \left|\psi_{1}-V_{\phi_{\varepsilon}}\right|<\varepsilon /(\|A y\|+\|y\|) \\
& \left|\psi_{2}-U_{\phi_{\varepsilon}}\right|<\varepsilon /(\|A y\|+\|y\|) .
\end{aligned}
$$

Consequently

$$
\begin{aligned}
& \left|[A y, z]-\left[y, A^{*}\left(z-\psi_{1}\right)-\psi_{2}\right]\right| \leqq \\
& \left|\left[A y, \psi_{1}-V_{\phi_{\varepsilon}}\right]+\left[y, \psi_{2}-U_{\phi_{\varepsilon}}\right]\right| \leqq \varepsilon
\end{aligned}
$$

(recall that $\left[A y, V_{\phi_{\varepsilon}}\right]+\left[y, U_{\dot{\phi}_{\varepsilon}}\right]=0$ for $y$ in $D\left(A_{H}\right)$. It follows from (3.7) that

$$
[A y, z]-\left[y, \widetilde{A}_{H}^{+} z\right]=0,
$$

proving that

$$
\widetilde{A}_{H}^{+} \supset A_{H}^{*}=\bar{A}_{H}^{+} .
$$

Corollary 3.7. $\widetilde{A}_{H}^{+*}=A_{H}$.

Proof. Immediate from Theorem 3.6 and the fact that $\widetilde{A}_{H}^{+}=\bar{A}_{H}^{+}$.

Corollary 3.8. If $R(H)$ is a Banach space $A_{H}^{+}=\widetilde{A}_{H}^{+}=A_{H}^{*}$.

Proof. By Theorem 2.3 $R\left(H^{*}\right)$ is closed and the assertion is immediate from Definition 3.1.

If $X$ and $Y$ are Hilbert spaces $G\left(-A^{*}\right)$ is trivially complemented. But almost nothing seems known about this concept in other spaces. (It is not even clear for example if the fact that $G(A)$ is complemented implies that $G\left(-A^{*}\right)$ is complemented.) We can however demonstrate the following sufficient condition that $G\left(-A^{*}\right)$ be complemented in reflexive Banach spaces.

THEOREM 3.9. If $A$ is a generalized Fredholm operator; i.e., $N(A)$ and $R(A)$ are complemented spaces in $X$ and $Y$, then $G\left(-A^{*}\right)$ is complemented.

Proof. It is well known (e.g., [4]) that if $A$ is a generalized Fredholm operator then so is $A^{*}$ and that the class of generalized 
Fredholm operators is equivalent to the class of operators admitting a generalized inverse, in other words a bounded linear operator $A^{+}: Y \rightarrow X$ satisfying the relations

$$
\begin{gathered}
A A^{+} A=A \\
A^{+} A A^{+}=A^{+} .
\end{gathered}
$$

Hence there exists a generalized inverse $A^{*+}$ for $A^{*}$ - in fact $A^{*+}=A^{+*}$. Let $J$ be the operator defined by $J(x)=-x$. Define $\widetilde{A}^{*+}: Y^{*} \rightarrow X^{*}$ by

$$
\widetilde{A}^{*+}=A^{*+} J A^{*} A^{*+}
$$

Now

$$
\begin{aligned}
\left(-A^{*}\right) \tilde{A}^{*+}\left(-A^{*}\right)(x) & =J A^{*} A^{*+} J A^{*} A^{*+} J A^{*}(x) \\
& =J A^{*} A^{*+} J^{2} A^{*}(x) \\
& =-A^{*} x .
\end{aligned}
$$

Also

$$
\begin{aligned}
\widetilde{A}^{*+}\left(-A^{*} \widetilde{A}^{*+}\right) & =A^{*+} J A^{*} A^{*+} J A^{*} A^{*+} J A^{*} A^{*+} \\
& =A^{*+} J A^{*} A^{*+} J^{2} A^{*} A^{*+} \\
& =\widetilde{A}^{*+}
\end{aligned}
$$

Thus $-A^{*}$ is a generalized Fredholm operator. Let $Q$ be a projection on $R\left(-A^{*}\right)$ (e.g., $\left.-A^{*} \widetilde{A}^{*+}\right)$. Let $S$ be a projection on $N\left(-A^{*}\right.$ ) $N\left(A^{*}\right)$. Define $P: Y^{*} \times X^{*} \rightarrow G\left(-A^{*}\right)$ by

$$
P\left(y^{*}, x^{*}\right)=\left((I-S) \widetilde{A}^{*+} Q x^{*}+S y^{*}, Q x^{*}\right) .
$$

$P$ is obviously continuous and onto since

$$
P\left(y^{*},-A^{*} y^{*}\right)=\left(y^{*},-A^{*} y^{*}\right) \text {. }
$$

Furthermore

$$
\begin{aligned}
P^{2}\left(y^{*}, x^{*}\right) & =P\left((I-S) \widetilde{A}^{*+} Q x^{*}+S y^{*}, Q x^{*}\right) \\
& =\left((I-S) \widetilde{A}^{*+} Q^{2} x^{*}+O+S^{2} y^{*}\right) \\
& =P\left(y^{*}, x^{*}\right) .
\end{aligned}
$$

The inconvenience of Theorem 3.9 is that it requires that $R(A)$ be closed at least if $Y$ is a general Banach space. Since we do not know any other sufficient condition, it seems worthwhile to explore ways in which the hypothesis that $G\left(-A^{*}\right)$ be complemented can be weakened. We devote the remainder of this section to this task.

Then next theorem and its two corollaries are generalizations of Theorem 3.9; while Theorem 3.13 and Corollary 3.14 represent a 
new approach.

THEOREM 3.10. If $G(-A+\lambda I)^{*}$ is complemented for some $\lambda$ then $A_{H}^{*}=\widetilde{A}_{H}^{+}$.

Proof. Let $A_{\lambda}:=A+\lambda I$. Then by Theorem $3.9 A_{\lambda H}^{*}=\widetilde{A}_{\lambda H}^{+}$. But $A_{\lambda H}^{*}=A_{H}^{*}+\bar{\lambda} I$. Therefore

$$
A_{H}^{*}=\widetilde{A}_{\lambda H}^{+}-\bar{\lambda} I .
$$

Now

$$
\begin{aligned}
{[H y, \phi] } & =\left[A y+\lambda y, V_{\lambda \phi}\right]+\left[y, U_{\lambda \phi}\right] \\
& =\left[A y, V_{\phi}\right]+\left[y, U_{\phi}\right] .
\end{aligned}
$$

Hence

$$
0=\left[A y, V_{\lambda \phi}-V_{\phi}\right]+\left[y, \bar{\lambda} V_{\lambda \phi}+U_{\lambda \phi}-U_{\phi}\right] .
$$

And so

$$
\begin{aligned}
& V_{\lambda \phi}-V_{\phi} \in D\left(A^{*}\right) \\
& V_{\lambda \phi}+U_{\lambda \phi}-U_{\phi}=A^{*}\left(V_{\phi}-V_{\lambda \phi}\right) .
\end{aligned}
$$

From (3.9) we conclude that

$$
D_{\lambda}^{+}=D^{+} .
$$

Further

$$
\begin{aligned}
A_{\lambda H}^{+}(z):= & A^{*}\left(a-V_{\lambda \phi}\right)+\bar{\lambda}\left(z-V_{\lambda \phi}\right)-U_{\lambda \phi} \\
= & A^{*}\left(z-V_{\lambda \phi}+V_{\phi}-V_{\phi}\right) \\
& \quad+\bar{\lambda}\left(z-V_{\lambda \phi}+V_{\phi}-V_{\phi}\right)-U_{\lambda \phi} \\
= & A^{*}\left(z-V_{\phi}\right)+A^{*}\left(V_{\phi}-V_{\lambda \phi}\right) \\
& \quad+\bar{\lambda}\left(z-V_{\phi}\right)+\bar{\lambda}\left(V_{\phi}-V_{\lambda \phi}\right)-U_{\lambda \phi} \\
= & A^{*}\left(z-V_{\phi}\right)+\bar{\lambda} V_{\lambda \phi}+U_{\lambda \phi}-U_{\phi} \\
& \quad+\bar{\lambda}\left(z-V_{\phi}\right)+\bar{\lambda} V_{\phi}-\bar{\lambda} V_{\lambda \phi}-U_{\phi}-U_{\lambda \phi} \\
= & A^{*}\left(z-V_{\phi}\right)+\bar{\lambda} z-U_{\phi} \\
= & A_{H}^{+} z+\bar{\lambda} z .
\end{aligned}
$$

Since

$$
\overline{A_{H}^{+}+\lambda I}=\bar{A}_{H}^{+}+\bar{\lambda} I,
$$

it follows from Theorem 3.9 that $\widetilde{A}_{\lambda H}^{+}=\widetilde{A}_{H}^{+}+\bar{\lambda} I$. By (3.8)

$$
A_{H}^{*}=\widetilde{A}_{H}^{+} \text {. }
$$

CoROLLARY 3.11. If $A+\lambda I$ is Fredholm or a generalized Fredholm operator (with $X, Y$ reflexive) then 


$$
A_{H}^{*}=\widetilde{A}_{H}^{+} \text {. }
$$

Corollary 3.12. Suppose $A$ is a differential operator. Then if $A$ has a nonempty essential resolvent

$$
A_{H}^{*}=\widetilde{A}_{H}^{+} \text {. }
$$

Proof. If $A$ has nonempty essential resolvent $\rho(A) A+\lambda I$ is Fredholm for $\lambda \in \rho(A)$ cf. [8] Ch. VI.

THEOREM 3.13. Suppose $N\left(A^{*}\right)$ is complemented in $Y^{*}$ and $H=M \circ A$. Assume that $\overline{D(M)^{\perp}}$ is complemented in $Y^{*}$ and that $D\left(M^{*}\right)$ is total over $F$. Define $A_{H}^{+}, \widetilde{A}_{H}^{+}$as in Definition 3.1 (taking $\left.V_{\phi} \in M^{*}(\phi), U_{\phi}=0\right)$. Then

$$
A_{H}^{*}=\widetilde{A}_{H}^{+}=\bar{A}_{H}^{+} .
$$

Proof. It is readily verified that $A_{H}^{+*}=A_{H}$ and that $A_{H}^{+}(z)$ is independent of the choice $V_{\phi}$ in $M^{*}(\phi)$. It remains to check that $A_{H}^{+}=\widetilde{A}_{H}^{+}$. Since the technique is the same as in the proof of Theorem 3.6 we only sketch the main steps. Note first that

$$
G\left(A^{*}\right)+\left(P R\left(M^{*}\right), 0\right)
$$

where $P$ is the projection on $N\left(A^{*}\right)$ is a direct sum. Let $\left\langle z_{n}\right\rangle$ be net converging weak* to $z$ in $D_{H}^{+}$. Let $\left\langle\beta_{n}\right\rangle$ be a net such that $\beta_{n} \in A_{H}^{+}$and $\beta_{n}$ converges $\beta$ weak ${ }^{*}$ to $\beta$. Let $Q$ be the projection of $Y^{*}$ onto $\left.\overline{\left(D(M)^{\perp}\right.}\right)^{\prime}$. Then $Q M_{\phi}^{*} \in M^{*}(\phi)$ and is an admissible $V_{\phi}$. Now

$$
\left(z_{n}, \beta_{n}\right)=\left(z_{n}-(I-P) Q M_{\phi_{n}}^{*}-P Q M_{\phi_{n}}^{*}, \beta_{n}\right)+\left(P Q M_{\phi_{n}}^{*}, 0\right) .
$$

Since the first term of this expression is in $G\left(A^{*}\right)$ it follows by (3.10) that

$$
P Q M_{\phi_{n}}^{*}=R\left(z_{n}, \beta_{n}\right)
$$

where $R$ is a continuous projection in $X$ to $\left(\left(N A^{*}\right)^{\prime}, 0\right)$. Therefore the nets $\left\langle P Q M_{\phi_{n}}^{*}\right\rangle$ and $\left\langle(I-P) Q M_{\phi_{n}}^{*}\right\rangle$ converge weak ${ }^{*}$. Hence $Q K_{\phi_{n}}^{*} \rightarrow \psi$ in $\overline{R\left(M^{*}\right)}$.

Corollary 3.14. Suppose $A$ is 1-1 and $N\left(A^{*}\right)$ is complemented. Let $A^{+}: Y \rightarrow X$ satisfy $A^{+} A=I$. Assume further that $\overline{\overline{D\left(H A^{+}\right)^{\perp}}}$ is complemented and $D\left(H A^{+}\right)^{*}$ is total over $F$. Let $V_{\phi} \in\left(H A^{+}\right)^{*}(\phi), U_{\phi}=0$. Then 


$$
A_{H}^{*}=\tilde{A}_{H}^{+} .
$$

Proof. Let $M=H A^{+}$. Then the hypotheses of Theorem 3.13 are satisfied.

Corollary 3.14 shows that in the case of 1-1 operators $A$ the hypothesis that $G\left(-A^{*}\right)$ is complemented can be replaced by weaker conditions. Moreover $\mathrm{HA}^{+}$and $\left(H A^{+}\right)^{*}$ are usually easy to calculate. ( $A^{+}$can often by identified with a Green's function.)

The following example is intended to illustrate some of the ideas in this section with special reference to Corollary 3.14.

ExAmple 3.15. Let $A: L^{1}[0, \infty) \rightarrow L^{1}[0, \infty)$ be given by $y^{\prime \prime}$ on

$D:=\left\{y \in L^{1}[0, \infty): y(0)=y^{\prime}(0)=0 ; y^{\prime}\right.$ is absolutely continuous and $\left.y^{\prime \prime} \in L^{1}[0, \infty)\right\}$.

Let $F$ be the space of bounded sequences $C$. Let $F^{+}$be the space $C_{00}$ of sequences with finitely many nonzero terms. Define a pairing on $C \times C_{00}$ by

$$
[\alpha, \beta]=\sum \alpha_{i} \beta_{i}, \quad \alpha \in C, \beta \in C_{00} .
$$

Then $F^{+}$is total. Under the weak and weak* topologies $F$ and $F^{+}$ are l.c.t.v.s such that $F^{*}=F^{+}$and $F^{+*}=F$.

Define $H: D \rightarrow F$ by

$$
\begin{aligned}
H y: & =\langle y(n)\rangle . \\
A^{+}: & =\int_{0}^{t}(t-s)(\cdot) d s .
\end{aligned}
$$

$A$ is obviously $1-1$. It is known see [8] (Ch. VI) that $A^{*}: L^{\infty}[0, \infty) \rightarrow$ $L^{\infty}[0, \infty)$ is given by $z^{\prime \prime}$ on

$$
\begin{aligned}
D^{*}:= & \left\{z \in L^{\infty}[0, \infty): z^{\prime}\right. \text { is abs. cont. and } \\
& \left.z^{\prime \prime} \in L^{\infty}[0, \infty) ; \lim _{t \rightarrow \infty} \overline{z(t) y^{\prime}}(t)-\overline{z(t)^{\prime} y}(t)=0, \quad y \in D\right\} .
\end{aligned}
$$

Then on $R(A) A^{+} A=I$ and

$$
H A^{+} w=\left\langle\int_{0}^{n}(n-s) w(s) d s\right\rangle, \quad w \in R(A) .
$$

Since $N\left(A^{*}\right)$ is finite dimensional it is complemented. Also

$$
{\overline{D\left(H A^{+}\right)^{*}}}^{*}=\overline{R(A)^{\perp}}=N\left(A^{*}\right) .
$$

This discussion shows that the hypotheses of Corollary 3.14 are satisfied. It is easily verified that $D\left(H A^{+}\right)^{*}=C_{00}$ and that $R\left(H A^{+}\right)^{*}$ consists of the space of piecewise linear functions of compact sup- 
port with corners on a finite subset of $\boldsymbol{R}_{+}$. A simple limiting argument (see [3] $\S 5.5$ for similar reasoning) implies that $\overline{R\left(H A^{+}\right)}$ consists of piecewise linear functions in $L^{\infty}[0, \infty]$ with infinitely many corners.

Application of Definition 3.1 and Corollary 3.14 now gives the following characterization of $A_{H}^{*}$.

$$
\begin{aligned}
D\left(A_{H}^{*}\right)= & \left\{z \in L^{\infty}[0, \infty): z^{\prime} \text { is abs. cont. on }(n, n+1), n \in Z_{+} ;\right. \\
& z^{\prime} \text { has arbitrary jumps on } Z_{+} ; \\
& \lim _{t \rightarrow \infty} \overline{(z(t)} y^{\prime}(t)-\left(z^{\prime}(t)+\sum_{n<t} \overline{\left(z^{\prime}\left(n^{+}\right)-z^{\prime}\left(n^{-}\right)\right)} y^{\prime}(t)=0\right), \\
& \left.y \in D A_{H}\right\} .
\end{aligned}
$$

$A_{H}^{*}$ is given by $z^{\prime \prime}$ on $D\left(A_{H}^{*}\right)$.

Note further that since

$$
[A y, z]-\left[y, A_{H}^{+} z\right]=[H y, \phi] .
$$

$H$ (and also $H A^{+}$): $D \rightarrow F$ is continuous if $D$ is given the graph topology and $F$ the weak topology defined above.

It is easy to show that $R(A)$ is dense and not surjective in $L^{\infty}[0, \infty)$. Hence by Theorem $2.3 R\left(A^{*}\right)$ is not closed. Further $R\left(A_{H}^{*}\right)=R\left(A^{*}\right)$. Applying the closed range theorem again we see that $R\left(A_{H}\right)$ is not closed either, so that the closure of $R(A)$ is not affected by the "perturbation" $H$. Obviously this fact can be generalized to give the following result.

CoROLlaRY 3.16. Let the hypotheses of Theorem 3.6 or Theorem 3.13 be satisfied then $R\left(A_{H}\right)$ is closed if and only if $R(A)$ is closed.

4. Extension theory. Suppose $A: X \rightarrow Y$ and $B: Y^{*} \rightarrow X^{*}$ are densely defined operators such that $B^{*} \supset A$. If $H: X \rightarrow F, K: Y^{*} \rightarrow G$ are boundary operators, for $A$ and $B$ then

$$
A_{H} \subset A \subset B^{*} \subset B_{K}^{*} .
$$

The purpose of this section is to determine the structure of all relations between $A_{H}$ and $B_{K}^{*}$.

We make the following assumptions concerning $A_{H}$ and $B_{K}$ :

(1) $N\left(A^{*}\right)$ and $N\left(B^{*}\right)$ are complemented spaces.

(2) $H=M \circ A, K=N \circ B$ where $D(M) \supset R\left(B^{*}\right)$ and $D(N) \supset R\left(A^{*}\right)$.

(3) $D\left(M^{*}\right)$ and $D\left(N^{*}\right)$ are total over $F$ and $G$.

(4) $\overline{D(M)^{\perp}}$ and $\overline{D(N)^{\perp}}$ are complemented spaces.

It follows from Theorem 3.13 that $A_{H}^{*}=\widetilde{A}_{H}^{+}$and $B_{K}^{*}=\widetilde{B}_{K}^{+}$. In $\widetilde{A}_{H}^{+}$ $\psi_{1} \in \overline{R\left(M^{*}\right)}$ and $\psi_{2}=0$. Similarly for $B_{K}^{+} \psi_{1} \in \overline{R\left(N^{*}\right)}, \psi_{2}=0$. (To avoid confusion we write " $\psi_{1}$ " in $\widetilde{B}_{K}^{+}$as " $\eta_{1}$ " when we are discussing 
$A_{H}^{*}$ and $B_{K}^{*}$ at the same time.)

We consider first the case when $\operatorname{dim} G\left(B_{K}^{*}\right) / G\left(A_{H}\right)<\infty$. Two preliminary lemmas will be required.

Lemma 4.1. Suppose $S: X \rightarrow C^{n}$ and $T: X \rightarrow C^{m}$ are operators such that $N(T) \supset N(S)$. Then $T=M S$ where $M$ is a $m \times n$ matrix. If furthermore the component functionals of $T$ are linearly independent $m \leqq n$ and $M$ is of full rank.

Proof. Let $\pi_{i}$ be the projection onto the $i$ th coordinate of $C^{n}$ or $\boldsymbol{C}^{m}$. Since

$$
\operatorname{ker} \pi_{i}(T) \supset N(T) \supset N(S)=\cap \operatorname{ker} \pi_{i}(S),
$$

it follows by Lemma 2.6 that

$$
\pi_{i}(T)=c_{i}{ }^{t} S, i=1, \cdots, m, c_{i}^{t} \in C^{n} .
$$

Choose $M$ to be the matrix

$$
\left(\begin{array}{c}
c_{1}^{t} \\
\vdots \\
c_{m}^{t}
\end{array}\right)
$$

Suppose the component functionals $\pi_{i}(T)$ are linearly independent. If the rows of $M$ are not linearly independent there exists $d \in \boldsymbol{C}^{m}$ such that $d^{t} M=0$. Hence

$$
d^{t} T=d^{t}(M S)=\left(d^{t} M\right) S=0,
$$

contradicting the independence of the component functionals of $T$. Thus rank $M=m$ and since row rank = column rank $m \leqq n$.

LEMmA 4.2. (A generalized Green's identity). Suppose that $\mathscr{A}: X \rightarrow Y, \mathscr{B}: Y^{*} \rightarrow X^{*}$ are relations such that $\mathscr{A} \subset \mathscr{B}^{*}$ and

$$
\operatorname{dim} G\left(\mathscr{A}^{*}\right) / G(\mathscr{B})=G\left(\mathscr{B}^{*}\right) / G(\mathscr{A})=n<\infty .
$$

Then there exist an $n \times n$ nonsingular matrix $\mathfrak{B}$ and continuous operators $\mathscr{J}: G\left(\mathscr{B}^{*}\right) \rightarrow C^{n} . \quad \tilde{J}: G\left(\mathscr{A}^{*}\right) \rightarrow C^{n}$ with linearly independent coordinate functionals such that

$$
\left[\mathscr{B}^{*} y, z\right]-\left[y, \mathscr{A}^{*} z\right]=\tilde{J}\left(z, \mathscr{A}^{*} z\right)^{*} \mathfrak{B} \mathscr{J}\left(y, \mathscr{B}^{*} y\right)
$$

on $G\left(\mathscr{A}^{*}\right) \times G\left(\mathscr{B}^{*}\right)$. Moreover in a Hilbert space setting $(X=Y$ a Hilbert space) $\mathscr{B}^{*}=\mathscr{A}^{*}$, and $\mathscr{A}$ symmetric then $\mathfrak{B}$ is skewhermitian. 
Proof. By Lemma $2.5 G(\mathscr{B})$ is the nullspace of a functional $\tilde{J}: G\left(\mathscr{A}^{*}\right) \rightarrow\left(G(\mathscr{B})^{\perp}\right)^{*}$. Now

$$
\left(G\left(\mathscr{A}^{*}\right) / G(\mathscr{B})\right)^{*} \cong G(\mathscr{B})^{\perp} .
$$

By (4.1)

$$
\left(G\left(\mathscr{A}^{*}\right) / G(\mathscr{B})\right)^{* *} \cong\left(G\left(\mathscr{A}^{*}\right) / G(\mathscr{B})\right)^{*} \cong C^{n} .
$$

Hence $\left(G(\mathscr{B})^{\perp}\right)^{*} \cong C^{n}$. The components of $\tilde{J}$ must be independent; for otherwise it would be equivalent to a functional with range of dimension $<n$. The existence of $\mathscr{J}$ follows by a similar argument.

Fix an element $(\alpha, \beta)$ in $G\left(\mathscr{A}^{*}\right)$. Then

$$
\left[\mathscr{B}^{*} y, \alpha\right]-[y, \beta]
$$

determines a functional whose nullspace contains $N(\mathscr{J})$. By Lemma 4.1 there exists $k(\alpha, \beta) \in C^{n}$ such that

$$
\left[\mathscr{B}^{*} y, \alpha\right]-[y, \beta]=k(\alpha, \beta)^{*} \mathscr{J}\left(y, \mathscr{B}^{*} y\right) .
$$

Since the component functionals of $\mathscr{J}$ are independent $k(\alpha, \beta)$ is unique. A simple calculation verifies that $k: G\left(\mathscr{A}^{*}\right) \rightarrow C^{n}$ is linear. If $\tilde{J}(\alpha, \beta)=0, k(\alpha, \beta)^{*} \mathscr{J}\left(y, \mathscr{A}^{*} y\right)=0$ on $G\left(\mathscr{A}^{*}\right)$. Since $\tilde{\mathcal{J}}$ is onto, $k(\alpha, \beta)=0$. Hence $N(\tilde{\mathscr{J}}) \subset N(k)$. Applying Lemma 4.1 again we find that $k(\alpha, \beta)=\mathfrak{B} \widetilde{\mathscr{J}(}(\alpha, \beta)$ where $\mathfrak{B}$ is a $n \times n$ nonsingular matrix.

We now show that $\mathscr{B}$ is skew-hermitian if $\mathscr{A}=\mathscr{B} \subset \mathscr{A} *$ and $\mathscr{A}$ is defined on a Hilbert space $H$. To see this note that (4.1) becomes

$$
\left[\mathscr{A}^{*} y, z\right]-\left[y, \mathscr{A}^{*} z\right]=\mathscr{J}\left(z, \mathscr{A}^{*} z\right) \mathfrak{B} \mathscr{J}\left(y, \mathscr{A}^{*} y\right)
$$

Taking congugate transposes and interchanging $y$ and $z$ gives

$$
\left[y, \mathscr{A}^{*} z\right]-\left[\mathscr{A}^{*} y, z\right]=\mathscr{J}\left(z, \mathscr{A}^{*} y\right] \mathfrak{B}^{*} \mathscr{J}\left(y, \mathscr{A}^{*} y\right) \text {. }
$$

Hence

$$
\mathscr{J} * \mathfrak{B} \mathscr{J}+\mathscr{J} * \mathfrak{B}^{*} \mathscr{J}=0
$$

which implies that $\mathfrak{B}^{*}=-\mathfrak{B}$.

REMARK 4.3. Note that if $\mathscr{A}$ and $\mathscr{B}$ are Fredholm operators then (4.1) is always true; for if $\kappa$ denotes the index of an operator, then

$$
G\left(\mathscr{A}^{*}\right) / G(\mathscr{B})=D\left(\mathscr{A}^{*}\right) / D(\mathscr{B})=\kappa\left(\mathscr{A}^{*}\right)-\kappa(\mathscr{B})
$$




$$
\begin{aligned}
& =-\kappa(\mathscr{A})+\kappa\left(\mathscr{B}^{*}\right)=D\left(\mathscr{B}^{*}\right) / D\left(\mathscr{A}^{*}\right) \\
& =G\left(\mathscr{B}^{*}\right) / G(\mathscr{A}) .
\end{aligned}
$$

(See [8] Theorem IV. 2.3.) (4.2) also holds if $\mathscr{A}$ and $\mathscr{B}$ have a nonempty Fredholm resolvent. This may be demonstrated by reasoning similar to Corollary 3.11.

Suppose

$$
\operatorname{dim} D\left(B^{*}\right) / D(A)=D\left(A^{*}\right) / D(B)=n .
$$

By Lemmas 2.5 and $4.2 A=B_{J}^{*}$ and $B=A_{\widetilde{J}}^{*}$ where $J, \widetilde{J}$ are boundary operators with range $C^{n}$. Since $A^{*}$ and $B^{*}$ are operators $J$ and $\widetilde{J}$ can be viewed as continuous operators with respect to the graph norms on $D\left(B^{*}\right)$ and $D\left(A^{*}\right)$. Thus we will write $J y$ instead of $J(y, A y)$.

If $z \in \widetilde{D}_{H}^{+}$and $\psi_{z} \in \psi(z)$ we write $\bar{z}$ for $z+\psi_{z}$. Similarly if $y \in \widetilde{D}_{K}^{+} \bar{y}$ means $y+\eta_{y}$ for some $\eta_{y} \in \eta(y)$. In terms of this notation we have the following "generalized Green's identity".

Lemma 4.4. For all $y$ in $\widetilde{D}_{K}^{+} z$ in $\widetilde{D}_{H}^{+}, \eta_{y}$, and $\psi_{z}$

$$
\left[\widetilde{B}_{k}^{+} y, z\right]-\left[y, \widetilde{A}_{I I}^{+} z\right]=(\widetilde{J} \bar{z})^{*} \mathfrak{B} J \bar{y}+\left[\eta_{y}, A^{*} \bar{z}\right]-\left[B^{*} \bar{y}, \psi_{z}\right] .
$$

Proof. By Lemma 4.2

$$
\left[\widetilde{B}_{k}^{+} y, \bar{z}\right]-\left[\bar{y}, \widetilde{A}_{I I}^{+} z\right]=(\widetilde{J} \bar{z})^{* \mathfrak{B} J \bar{y}}
$$

since $\widetilde{B}_{k}^{+} y:=B^{*} \bar{y}$ and $\widetilde{A}_{H}^{+} z=A^{*} \bar{z}$. Adding $\left[\eta_{y}, A^{*} \bar{z}\right]-\left[B^{*} \bar{y}, \psi_{z}\right]$ to both sides gives the result.

THEOREM 4.5. Suppose $\mathscr{A}, \mathscr{B}$ are relations such that $G(\mathscr{B}) /$ $G(\mathscr{A})=n<\infty$. Then $\mathscr{A} \subset \mathscr{C} \subset \mathscr{B}$ if and only if there exists a $k \times n(k<n)$ matrix $\mathscr{D}$ of full rank such that

$$
G(\mathscr{C})=N(\mathscr{D} \mathscr{J}) \cap G(\mathscr{B})
$$

where $\mathscr{J}$ is a boundary operator for . .

Proof. Suppose $\mathscr{A} \subset \mathscr{C} \subset \mathscr{B}$. Then $G(\mathscr{C})$ is the nullspace of some nonzero boundary operator $\mathscr{H}: G(\mathscr{B}) \rightarrow C^{k}, k \leqq n$. Since $N(\mathscr{H}) \supset N(\mathscr{J}) \mathscr{H}=\mathscr{D} \mathscr{J}$ by Lemma 4.1 where $\mathscr{D}$ is a $k \times \eta$ matrix of full rank. The converse is trivial since $\mathscr{D} \mathscr{J}$ is a boundary operator.

It is sometimes convenient to give a "parametric" rather than a boundary operator description of extensions $\mathscr{C}$ between $\mathscr{A}$ and $\mathscr{B} *$. 
CoRollary 4.6. $\mathscr{C}$ is an relation between $\mathscr{A}$ and $\mathscr{B}^{*}$ if and only if there exists a subspace $S_{c}$ of $C^{n}$ such that

$$
\bar{D}(\mathscr{C})=\left\{y \in D\left(\mathscr{B}^{*}\right):[\mathscr{J}(y, \mathscr{B} y), \phi]=0 ; \phi \in S_{c}\right\} \text {. }
$$

Proof. Let $S_{c}=R\left(\mathscr{D}^{*}\right)$.

We now turn to the description of $\mathscr{C}^{*}$. We introduce the following notation if $S$ is a finite dimensional space let $(S)$ signify a matrix whose columns form a basis of $S$.

THEOREM 4.7. If $\mathscr{A} \subset \mathscr{C} \subset \mathscr{B}$ then $\mathscr{B}^{*} \subset \mathscr{C}^{*} \subset \mathscr{A}^{*}$ and

$$
G\left(\mathscr{C}^{*}\right)=\left\{\left(y, \mathscr{A}^{*} y\right):(N(\mathscr{D}))^{*} \mathfrak{B}^{*} \widetilde{J}\left(y, \mathscr{A}^{*} y\right)=0\right\} \text {. }
$$

Proof. We consider only the last statement. Let $\left(\alpha, \mathscr{A}^{*} \alpha\right) \in$ $G\left(\mathscr{C}^{*}\right)$. Then by Lemma $4.1 \tilde{J}^{*}(\alpha \mathscr{L} \alpha)$ is a functional on $G\left(\mathscr{\mathscr { A }}^{*}\right)$ whose nullspace includes $N(\mathscr{D} \mathscr{J}(\cdot))$. Hence on all of $G\left(\mathscr{A}^{*}\right)$

$$
\tilde{J}^{*}\left(\alpha, \mathscr{A}^{*} \alpha\right) \mathfrak{B} \mathscr{J}=\phi^{*} \mathscr{D} \mathscr{J}
$$

where $\phi \in C^{n}, k \leqq n$. This implies

$$
\mathfrak{B}^{*} \tilde{J}\left(\alpha, \mathscr{A}^{*} \alpha\right) \in R\left(\mathscr{D}^{*}\right) \text {. }
$$

Equivalently

$$
(N(\mathscr{D}))^{*} \mathfrak{B}^{*} \tilde{\mathcal{J}}\left(\alpha, \mathscr{A}^{*} \alpha\right)=0 .
$$

On the other hand if $(\alpha, \mathscr{A} \alpha)$ satisfies (4.4)

$$
\begin{aligned}
\left(\tilde{J}^{*}(\alpha, \mathscr{A} \alpha) \mathfrak{B}_{\mathscr{J}}(y, \mathscr{B} y)\right)^{*} & =\mathscr{J}^{*}\left(y, \mathscr{B}^{*} y\right) \mathfrak{B}^{*} \tilde{\mathscr{J}}\left(\alpha, \mathscr{A}^{*} \alpha\right) \\
& =\mathscr{J}^{*}\left(y, \mathscr{B}^{*} y\right) \mathscr{D}^{*} \phi \\
& =\left(\mathscr{D} \mathscr{J}\left(y, \mathscr{B}^{*} y\right)\right)^{*} \phi \\
& =0 .
\end{aligned}
$$

So that $\left(\alpha, \mathscr{A}^{*} \alpha\right) \in G\left(\mathscr{C}^{*}\right)$.

Corollary 4.8 .

$$
G\left(\mathscr{C}^{*}\right)=\left\{\left(y, \mathscr{A}^{*} y\right):\left[\mathfrak{B}^{*} \mathscr{J}\left(y, \mathscr{A}^{*} y\right), \theta\right]=0 \text { for all } \theta \in S_{c}^{*}\right\} \text {. }
$$

COROLlaRY 4.9. Suppose $\mathscr{A}$ is a symmetric relation (i.e., $\left.\mathscr{A} \subset \mathscr{A}^{*}\right)$ defined on a Hilbert space $\mathscr{H}$ and

$$
\operatorname{dim} G\left(\mathscr{A}^{*}\right) / G(\mathscr{A})=n<\infty .
$$

Let $\mathcal{F}$ be the boundary operator for $\mathscr{A}$. Then $\mathscr{A} \subset \mathscr{C} \subset \mathscr{A}^{*}$ is 
self-adjoint if and only if there exists a $k \times n(k<n)$ matrix of full rank $\mathscr{D}$ such that

$$
\mathscr{D}=(N(\mathscr{D}))^{*} \mathfrak{B} *
$$

or equivalently

$$
\mathscr{D} \mathfrak{B}^{-1} \mathscr{D}^{*}=0
$$

where $\mathfrak{B}$ is the skew-hermitian matrix of Lemma 4.2 .

Proof. Apply Theorems 4.5 and 4.7. It is clear that rank $\mathscr{D}$ must be less than $n$.

If we can find boundary operators $\mathscr{J}, \tilde{J}$ determining $A_{H}$ and $B_{K}$ as restrictions of $B_{K}^{*}$ and $A_{H}^{*}$, Theorem 4.5 - Corollary 4.8 can be applied verbatim to determine all extensions between $A_{H}$ and $B_{K}^{*}$ and their adjoints. Let us assume that $D(H), D(J) \supset G\left(B_{K}^{*}\right)$. Then

$$
\begin{gathered}
\mathscr{J}:=\left(\begin{array}{c}
H \\
J \\
\widetilde{K}
\end{array}\right) \\
\tilde{J}:=\left(\begin{array}{c}
K \\
\widetilde{J} \\
\widetilde{H}
\end{array}\right)
\end{gathered}
$$

where $\widetilde{K}, \widetilde{H}$ are boundary operators determining $B^{*}$ and $A^{*}$ as restrictions of $B_{K}^{*}$ and $A_{H}^{*}$. Clearly the only novelty is the determination of $\widetilde{K}$ and $\widetilde{H}$.

Lemma 4.10. Suppose $R(K)=C^{k}$ and $R(H)=C^{n}$. Then

$$
\begin{aligned}
\widetilde{K}\left(y, B_{K}^{*} y\right) & =\left[\theta_{y},(R(N \circ B))\right] \\
\widetilde{H}\left(z, A_{H}^{*} z\right) & =\left[(R(M \circ A)), \psi_{z}\right] .
\end{aligned}
$$

Proof. By definition $K$ is an operator on $G\left(B_{K}^{*}\right)$ whose nullspace is exactly $G\left(B^{*}\right)$. If $e_{i}$ is the $i$ th row of $(R(N \circ B))$. Choose $z_{i} \in D(B)$ such that

$$
N \circ B z_{i}=e_{i}
$$

Define

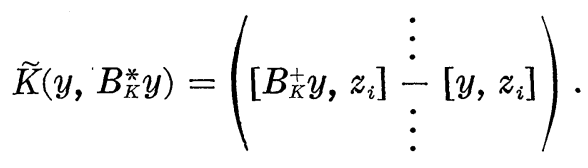


By Lemma 4.4

$$
\begin{aligned}
\widetilde{K}\left(y, B_{K}^{*} y\right) & =\left(\begin{array}{c}
\vdots \\
{\left[\eta_{y}, B z_{i}\right]} \\
\vdots
\end{array}\right) \\
& =\left(\begin{array}{c}
\left.\vdots \theta_{y}, \hat{N} \circ B z_{i}\right] \\
\vdots
\end{array}\right) \\
& =\left[\theta_{y},(R(N \circ B))\right] .
\end{aligned}
$$

(Note that $\tilde{K}$ is well defined since $\eta_{y}-\eta_{y}^{\prime} \in R(B)=N\left(B^{*}\right)$.) If

$$
\begin{aligned}
{\left[\theta_{y},(R(N \circ B))\right]=0, \text { then } \theta_{y} \in R(N \circ B) } & \Longleftrightarrow \eta_{y} \in R(B) \in N\left(B^{*}\right) \\
& \Longleftrightarrow\left(y, B_{K}^{*} y\right) \in G\left(B^{*}\right) .
\end{aligned}
$$

So that $N(\widetilde{K}) \subset G\left(B^{*}\right)$. The reverse inclusion is trivial. This proves (4.5). The proof of (4.6) is similar and will be omitted.

TheOREM 4.11. If $C$ is a closed relation between $A_{H}$ and $B_{K}^{*}$ then

$$
G\left(C^{*}\right)=N\left[(N[(\mathscr{D}))]^{*} \mathfrak{B}^{*}\left(\begin{array}{c}
\bar{H} \\
\widetilde{J} \\
K
\end{array}\right) \cap G\left(A_{H}^{*}\right)\right.
$$

Equivalently

$$
D\left(C^{*}\right)=\left\{z: \mathfrak{B}\left(\begin{array}{c}
\tilde{H} \\
\widetilde{J} \\
K
\end{array}\right) \in S_{c}=R\left(\mathscr{D}^{*}\right)\right\}
$$

where $\mathfrak{B}$ is the $n+m+k \times n+m+k$ nonsingular matrix given in Lemma 4.2 taking

$$
\begin{aligned}
& " \mathscr{A}^{* \prime \prime}=A_{H}^{*} \quad " \mathscr{B}^{* \prime \prime}=B_{K}^{*} \\
& " \widetilde{J}^{\prime \prime}=\left(\begin{array}{c}
\tilde{K} \\
J \\
H
\end{array}\right) " \mathscr{J}^{\prime \prime}=\left(\begin{array}{c}
\tilde{H} \\
\widetilde{J} \\
K
\end{array}\right) .
\end{aligned}
$$

EXAMPLE 4.12. For fixed $1 \leqq p \leqq \infty$ let

$$
\begin{aligned}
& W^{1, p}[a, b]:=\left\{y \in y^{\prime} \text { is abs. cont., } y^{\prime} \in L^{p}[a, b]\right\}, \\
& W_{0}^{1, p}[a, b]:=\left\{y \in W^{1, p}[a, b]: y(a)=y(b)=0\right\} .
\end{aligned}
$$

Define $A$ on $W_{0}^{1, p}[0,1]$ by $A y:=-i y^{\prime}$ and $B$ on $W_{0}^{1, q}[0,1], 1 / p+1 / q=1$, 
by $B z:=-i z^{\prime}$. Then $A^{*}$ is given by $-i z^{\prime}$ on $W^{1, q}[a, b]$ and $B^{*}$ is given by $-i y^{\prime}$ on $W^{1, p}[a, b]$. Further $A \subset B^{*}$ and $B \subset A^{*}$. Let $G=$ $F=C$ and define

$$
H: W^{1, p}\left[0, \frac{1}{2}\right)+W^{1, p}\left(\frac{1}{2}, 1\right] \longrightarrow C \text { by } H y:=y\left(\frac{1}{2}+\right) .
$$

Similarly let $K: W^{1, q}\left[0, \frac{1}{2}\right)+W^{1, q}\left(\frac{1}{2}, 1\right] \rightarrow C$ be given by $k y=y\left(\frac{1}{2}^{-}\right)$. By the methods of $\S 3$ (cf. Example 3 ) it is readily shown that $A_{H}^{*}$ and $B_{K}^{*}$ are given by $-i z^{\prime},-i y^{\prime}$ on $W^{1, q}\left[0, \frac{1}{2}\right)+W^{1, q}\left(\frac{1}{2}, 1\right]$ and $W^{1, p}\left[0, \frac{1}{2}\right)+W^{1, p}\left[\frac{1}{2}, 1\right]$ respectively.

Clearly $\widetilde{K} y=y\left(\frac{1}{2}^{+}\right)-y\left(\frac{1}{2}^{-}\right)$and a boundary operator $\mathscr{J}$ defining $A_{H}$ as a restriction of $B_{K}^{*}$ is

$$
\mathscr{J} y=\left(\begin{array}{l}
y\left(+\frac{1}{2}\right)-y\left(-\frac{1}{2}\right) \\
y(0) \\
y(1) \\
y\left(\frac{1}{2}+\right)
\end{array} \mid\right.
$$

Similarly $B_{K} \subset A_{H}^{*}$ is determined by

$$
\tilde{J} z=\left(\begin{array}{l}
z\left(\frac{1}{2}^{+}\right)-z\left(\frac{1}{2}^{-}\right) \\
z(0) \\
z(1) \\
z\left(\frac{1}{2}\right)
\end{array}\right)
$$

A short calculation reveals that $\mathfrak{B}$ is the skew-Hermitian unitary martix

$$
\left(\begin{array}{rrrr}
0 & 0 & 0 & -i \\
0 & i & 0 & 0 \\
0 & 0 & -i & 0 \\
-i & 0 & 0 & 0
\end{array}\right)
$$

Thus if

$$
\mathscr{D}=\left(\begin{array}{llll}
1 & 0 & 1 & 0 \\
0 & 2 & 0 & 1
\end{array}\right)
$$

$N(\mathscr{D})$ is spanned by

$$
\left(\begin{array}{r}
1 \\
0 \\
-1 \\
0
\end{array}\right), \quad\left(\begin{array}{r}
0 \\
1 \\
0 \\
-1
\end{array}\right)
$$

and application of Theorem 4.7 gives the ajoint boundary conditions 


$$
\begin{aligned}
& -\left(z\left(\frac{1}{2}^{+}\right)-z\left(\frac{1}{2}^{-}\right)\right)-z(0)=0 \\
& -z(1)+z\left(\frac{1}{2}^{+}\right)=0
\end{aligned}
$$

for $C^{*}$.

If $G\left(B_{K}^{*}\right) / G\left(A_{H}\right)$ is not finite dimensional the foregoing extension theory breaks down because the linear dependence principle is not available.

We conclude this section with a new approach which works in the infinite dimensional case for extensions with closed range, and a new characterization of self-adjoint extensions in Hilbert space.

LeMma 4.12. Let $S$ be a subspace of $Y^{*}$ and $N_{c}$ be a closed subspace of $N\left(B^{*}\right)$. Let

$$
\begin{gathered}
D(\mathfrak{C}):=\left\{y \in\left(D\left(B^{*}\right) \cap N\left(B^{*}\right)^{\prime}\right)+N_{c}:\left[B^{*} y, \psi\right]=0,\right. \\
\psi \in S\} .
\end{gathered}
$$

Define $G(\mathbb{E})$ by $B^{*}$ on $D(\mathbb{E})$. Then (5 is closed.

Proof. If $y_{n} \rightarrow y$ and $B^{*} y_{n} \rightarrow z y \in D\left(B^{*}\right)$ and $z=B^{*} y$ since $B^{*}$ is closed. Further $\left[B^{*} y, \psi\right]=0 \quad \forall \psi \in S$ by the continuity of the pairing and $y$ must lie in the closed set $N\left(B^{\prime}\right)^{\prime}+N_{c}$.

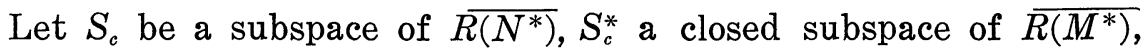
$N_{c}$ a closed subspace of $N\left(B^{*}\right)$ and $N_{c}^{*}$ a subspace of $N\left(A^{*}\right)$. Define

$$
\begin{gathered}
D(C):=\left\{y \in\left(D\left(B^{*}\right) \cap N\left(B^{*}\right)^{\prime}+N_{c}\right)-S_{c}:\right. \\
\left.B^{*} \bar{y} \perp S_{c}^{*}+N_{c}^{*}\right\} \\
G(C):=\left\{\left(y, B^{*} \bar{y}\right): y \in D(\mathfrak{S})\right\} .
\end{gathered}
$$

We call $C$ the relation determined by $S_{c}, S_{c}^{*} N_{c}$ and $N_{c}^{*}$. Clearly $A_{H} \subset C \subset B_{K}^{*}$ and

$$
G(C)=G(\mathbb{E})-\left(Q S_{c}, 0\right)
$$

where $\mathfrak{C} \subset B^{*}$ is defined relative to $N_{c}$ and $N\left(B^{*}\right)^{\prime}+N_{c}$ by (4.7) and $Q$ is a projection on $N\left(B^{*}\right)^{\prime}$. Since $G(\sqrt{5})$ is closed by Lemma 4.12 and (4.9) is equivalent to a direct sum, $C$ is closed. This proves the following result:

THEOREM 4.13. Let the hypotheses of Theorem 3.13 hold. Let $S_{c}, S_{c}^{*}, N_{c}$ and $N_{c}^{*}$ be subspaces of $\overline{R\left(N^{*}\right)}, \overline{R\left(M^{*}\right)}, N\left(B^{*}\right)$ and $N\left(A^{*}\right)$ such that $S_{c}$ and $N_{c}$ are closed. Then there exists a unique closed relation between $A_{H}$ and $B_{K}^{*}$ determined by $S_{c}, S_{c}^{*}, N_{c}$ and $N_{c}^{*}$.

The following is a partial converse to Theorem 4.13: 
THeOREM 4.14. Suppose $C$ is a closed relation between $A_{H}$ and $B_{K}^{*}$ then there exist closed subspaces $N_{c} \subset N\left(B^{*}\right), N_{c}^{*} \subset N\left(A^{*}\right), S_{c}^{*} \subset \overline{R\left(M^{*}\right)}$ and $S_{c} \subset \overline{R\left(N^{*}\right)}$ such that

$$
\begin{aligned}
& S_{c}=\psi: D(C)^{1} \\
& R(C)^{\perp}=S_{c}^{(*}+N_{c}^{*} \\
& N(C)=S_{c}+N_{c} .
\end{aligned}
$$

Moreover if $C$ has closed range $C$ is determined by $S_{c}, S_{c}^{*}, N_{c}$ and $N_{c}^{*}$.

Proof. Set $S_{c}^{*}:=R(C)^{\perp} \cap \overline{R\left(M^{*}\right)}, N_{c}^{*}:=R(C)^{\perp} \cap N\left(A^{*}\right), S_{c}:=N(C) \cap$ $\overline{R\left(N^{*}\right)}, N_{c}:=N(C) \cap N\left(B^{*}\right)$. Since $C \subset B_{K}^{*}, S_{c}=\psi: D(C)$. Clearly $R(C)^{\perp} \supset S_{c}^{*}+N_{c}^{*}$ and $N(C) \supset S_{c}+N_{c}$. However since $A_{H} \subset C$ and $C \subset B_{K}^{*}$

$$
\begin{aligned}
& R(C)^{\perp} \subset R\left(A_{H}^{*}\right)^{\perp}=N\left(\widetilde{A}_{H}^{+}\right)=\overline{R\left(M^{*}\right)}+N\left(A^{*}\right) \\
& N(C) \subset N\left(B_{K}^{*}\right)=\overline{R\left(N^{*}\right)} \neq N\left(B^{*}\right) .
\end{aligned}
$$

Applying the definitions of $S_{c}^{*}, N_{c}^{*}, S_{c}$ and $N_{c}$ gives the reverseinclusions. Now suppose $C$ has closed range. Let $C^{\prime}$ be the relation determined by $S_{c}^{*}, N_{c}^{*}, S_{c}$ and $N_{c}$ according to Theorem 4.13. Obviously $C^{\prime} \supset C$. Since

$$
R\left(C^{\prime}\right)^{\perp}=R(C)^{\perp}=S_{c}^{*}+N_{c}^{*}
$$

and $R(C)$ is closed, $\overline{R\left(C^{\prime}\right)}=R(C)$. Thus $R\left(C^{\prime}\right)=R(C)$. From (4.8) and (4.10) $N\left(C^{\prime}\right)=N(C)$. Let $(\alpha, \beta) \in G\left(C^{\prime}\right)$. Then there exists $\alpha^{\prime} \in$ $D(C)$ such that $\left(\alpha^{\prime}, \beta\right) \in G\left(C^{\prime}\right)$. Hence $\alpha-\alpha^{\prime} \in N\left(C^{\prime}\right)=N(C)$. Thus $\alpha \in D(C)$ and $\beta=C(\alpha)$.

THEOREM 4.15. If $C$ is a relation with closed range between $A_{H}$ and $B_{K}^{*}, C^{*}$ is a relation with closed range between $B_{K}$ and $A_{H}^{*}$ and $C^{*}$ is determined by $S_{c}=S_{c^{*}}^{*}, S_{c}^{*}=S_{c}, N_{c^{*}}^{*}=N_{c^{*}}$ and $N_{c^{*}}^{*}=N_{c}$.

Proof. We verify only the last statement

$$
\begin{aligned}
& S_{c^{*}}:=N\left(C^{*}\right) \cap \overline{R\left(M^{*}\right)}=R(C)^{\perp} \cap \overline{R\left(M^{*}\right)}:=S_{c}^{*} \\
& S_{c^{*}}:=R\left(C^{*}\right)^{\perp} \cap \overline{R\left(N^{*}\right)}=N(C) \cap \overline{R\left(N^{*}\right):}=S_{c} \\
& N_{c^{*}:}=N\left(C^{*}\right) \cap N\left(A^{*}\right)=R(C)^{\perp} \cap N\left(A^{*}\right)=N_{c}^{*} \\
& N_{c^{*}}^{*}:=R\left(C^{*}\right)^{\perp} \cap N\left(B^{*}\right)=N(C) \cap N\left(B^{*}\right)=N_{c} .
\end{aligned}
$$

The following result is an alternate characterization of $D\left(C^{*}\right)$ without the space $N_{c^{*}}$ that is available if $A$ is a finite dimensional 
restriction of $B^{*}$

Corollary 4.16. Suppose $\operatorname{dim} D\left(B^{*}\right) / D(A)<\infty$. Then

$$
\begin{aligned}
D\left(C^{*}\right)= & \left\{z \in \widetilde{D}_{H}^{+}: \psi_{z} \in S_{c}^{*} ; A^{*} \bar{z} \perp S_{c} ;\right. \\
& \left.(\widetilde{J} \bar{z})^{*} \mathfrak{B} J \bar{y}=0, \forall y \text { in } D(C)\right\} .
\end{aligned}
$$

Proof. If $z \in D\left(C^{*}\right), \psi_{z} \in S_{c}^{*}$ and $A^{*} \bar{z} \perp S_{c}$ by Theorem 4.15. If $y \in D(C)$, by (4.8) $\bar{y} \in D(\mathbb{E})$ for any $\eta_{y}$. Similarly by Theorem 4.15 and (4.8) if $z \in D\left(C^{*}\right) \bar{z} \in D\left(C^{*}\right)$. Since $C$ and $C^{*}$ are mutually adjoint, by Lemma $4.4(\widetilde{J} \bar{z})^{*} \mathfrak{B} J \bar{y}=0$ (taking $\eta_{\bar{y}}, \psi_{\bar{z}}=0$ ). Therefore $D\left(C^{*}\right)$ satisfies (4.11). Conversely if $z$ satisfies (4.11), application of Lemma 4.4 and the definitions of $S_{c}^{*}$ and $S_{c}$ gives

$$
\left[\widetilde{B}_{K}^{+} y, z\right]-\left[y, \widetilde{A}_{I I}^{+} z\right]=0
$$

for all $y$ in $D(C)$. Hence $z \in D\left(C^{*}\right)$.

REMARK 4.17. The analogue of (4.11) can in the same way be proved for $C$, i.e.,

$$
\begin{aligned}
D(C)= & \left\{y \in \widetilde{D}_{K}^{+}: \eta_{y} \in S_{c} ; B^{*} \bar{y} \perp S_{c}^{*}\right. \\
& \left.(\widetilde{J} \bar{z})^{*} \mathfrak{B} J \bar{y}=0, \forall z \in D\left(C^{*}\right)\right\} .
\end{aligned}
$$

We turn now to the characterization of self-adjoint extensions in a Hilbert space setting. Here the hypothesis that $C$ has closed range is no longer needed, the next two theorems give simple necessary and sufficient conditions for the existence of a rich supply of self-adjoint extensions between $A_{H}$ and $A_{H}^{*}$.

THEOREM 4.18. Let $A$ be a symmetric operator defined on a Hilbert space $H$. Then if $A$ has a self-adjoint extension $\sqrt{ }$, for each closed subspace $S$ of $\overline{R\left(M^{*}\right)}$ there exists a self-adjoint extension $C_{s}$ of $A_{H}$ such that

$$
\begin{aligned}
& D\left(C_{s}\right)=\left\{y \in D(\mathfrak{S})-S: A^{*} \bar{y} \perp S\right\} \\
& G\left(C_{s}\right)=\left\{\left(y, A^{*} \bar{y}\right): y \in D\left(C_{s}\right)\right\} .
\end{aligned}
$$

Proof. Let $A_{H} \subset C_{s} \subset A_{H}^{*}$ be the relation determined by (4.12). Then

$$
\begin{aligned}
{\left[C_{s} y, z\right]-\left[y, C_{s} z\right]=\left[A^{*} \bar{y}, \bar{z}\right]-\left[\bar{y}, A^{*} \bar{z}\right] } & \\
& -\left[A^{*} \bar{y}, \psi_{z}\right]+\left[\eta_{y}, A^{*} \bar{z}\right]
\end{aligned}
$$

where $\bar{y}=y+\eta_{y}, \bar{z}=z+\psi_{z}$ and $\eta_{y}, \psi_{z} \in S$. By (4.12) and the selfadjointness of $\sqrt{5}$ the right side of $(4.13)$ is zero, showing that $C_{s}$ is 
symmetric. Now suppose $(\alpha, \beta) \in G\left(C_{s}^{*}\right)$. Since $C_{s}^{*} \subset A_{I I}^{*}, \beta=A^{*} \bar{\alpha}$. Further

$$
\begin{aligned}
{\left[C_{s} y, \alpha\right]-\left[y, A^{*} \bar{\alpha}\right]=} & 0 \\
= & {\left[A^{*} \bar{y}, \bar{\alpha}\right]-\left[y, A^{*} \bar{\alpha}\right] } \\
& -\left[A^{*} \bar{y}, \psi_{\alpha}\right]+\left[\eta_{y}, A^{*} \bar{\alpha}\right] .
\end{aligned}
$$

Since

$$
\begin{aligned}
\psi_{\alpha} \in N\left(C_{s}^{*}\right) \cap \overline{R\left(M^{*}\right)} & =R\left(C_{s}\right)^{\perp} \cap \overline{R\left(M^{*}\right)} \\
& =S
\end{aligned}
$$

and

$$
\eta_{y} \in S \subset N\left(C_{s}\right)=R\left(C_{s}^{*}\right),
$$

the last two terms in (4.14) vanish and hence

$$
\left(\bar{\alpha}, A^{*} \bar{\alpha}\right) \in G\left(\varsigma^{*}\right)=G(\sqrt{5}) \text {. }
$$

Thus $\alpha \in D(5)-S$. Since $\eta_{y}$ is arbitrary in $S, A^{*} \bar{\alpha} \perp S$. We conclude that $(\alpha, \beta) \in G\left(C_{s}\right)$ and that $C_{s}=C_{s}^{*}$.

THEOREM 4.19. Let $A$ be a symmetric operator defined on a Hilbert space $\mathscr{H}$. Suppose $C$ is a self-adjoint extension of $A_{H}$. Then $A$ has a self-adjoint extension (5. Moreover if $S:=R(\sqrt{ }), C$ is the self-adjoint extension $C_{s}$ determined by $\mathbb{C}$ and $S$ given by Theorem 4.18.

Proof. Define c by

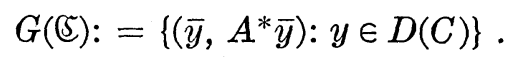

Obviously by $\mathbb{S} \supset A$. It follows at once from (4.13) that $\mathbb{E}$ is symmetric since $\eta_{y}, \psi_{z} \in N(C) \perp R(C)$. Suppose $\left(\bar{\alpha}, A^{*} \bar{\alpha}\right) \in G\left(\complement^{*}\right)$. Let $\psi \in \psi: D(C)=N(C)$. Then by (4.13) again

$$
\left[(C y, \bar{\alpha}-\psi]-\left[\bar{y}, A^{*} \bar{\alpha}\right]=\left[\eta_{y}, A^{*} \bar{\alpha}\right] .\right.
$$

Hence

$$
[C y, \bar{\alpha}-\dot{\psi}]-\left[y, A^{*} \bar{\alpha}\right]=0,
$$

so that

$$
\left(\bar{\alpha}-\psi, A^{*} \bar{\alpha}\right) \in G\left(C^{*}\right)=G(C) .
$$

We conclude that $\bar{\alpha}-\psi \in D(C),\left(\bar{\alpha}, A^{*} \bar{\alpha}\right) \in \mathfrak{C}$. Thus $\mathfrak{S}^{*} \subset \mathbb{C}$ and $\sqrt{ }$ is self-adjoint.

Since $R(\mathfrak{S})=R(C)$ and $R(C)^{\perp} \subset N\left(A_{H}^{*}\right)=\overline{R\left(M^{*}\right)}, S$ is a closed 
subspace of $\overline{R\left(M^{*}\right)}$. By Theorem 4.18 there exists a self-adjoint extension $C_{s}$ determined by $\left(5\right.$ and $S$. By (4.12) $C=C_{s}$.

CoRollary 4.20. Suppose $A$ is a symmetric operator on a Hilbert space $\mathscr{H}$ with equal deficiency indices. Let $R(H)$ be a Banach space and let the hypotheses of Corollary 3.14 be satisfied. Further let $S$ be a closed subspace of $R(H)$. Then $A_{H}$ has a selfadjoint extension determined by the boundary conditions

$$
\begin{array}{r}
{\left[H A^{+}\left(A^{*} \bar{z}\right), \phi\right]=0, \phi \in S} \\
\bar{z} \in \mathbb{S}
\end{array}
$$

where $(5)$ is a self-adjoint extension of $A$.

Proof. Since $A$ has equal deficiency indices there is a selfadjoint extension $\sqrt{5}$ of $A$. By Corollary 3.8 $A_{H}^{*}=A_{H}^{+}$. By Corollary $3.14 M=H A^{+}$. Now apply Theorem 4.18.

EXAMPLe 4.21. We use Corollary 4.20 to find self-adjoint extensions of $A_{H}$ in Example 4.12 when $p=2$. Here

$$
A^{+}=i \int_{0}^{T}(\cdot) d s
$$

on $L^{2}[0,1]$. Further

$$
\begin{aligned}
{\left[H A^{+} z, \phi\right] } & =\bar{\phi} i \int_{0}^{1 / 2} z d t \\
& =\int_{0}^{1} z i \lambda_{[0.1 / 2]}(t) \bar{\phi} d t
\end{aligned}
$$

so that $\left(H A^{+}\right)^{*} \phi=-i \lambda_{[0,1 / 2]}(t) \phi$. Since $\bar{z}=z+\left(H A^{+}\right)^{*} \phi$ is absolutely continuous $\bar{z}\left(\frac{1}{2}^{+}\right)=\bar{z}\left(\frac{1}{2}^{-}\right)$and we obtain $\phi=i\left(z\left(\frac{1}{2}^{+}\right)-z\left(\frac{1}{2}^{-}\right)\right)$. Moreover

$$
\bar{z}=\left\{\begin{array}{ll}
z+z\left(\frac{1}{2}^{+}\right)-. z\left(\frac{1}{2}-\right) & 0 \leqq t<\frac{1}{2} \\
z\left(\frac{1}{2}^{+}\right) & t=\frac{1}{2} \\
z & t>\frac{1}{2}
\end{array}\right\},
$$

and

$$
\begin{aligned}
H A^{+} A^{*} \bar{z} & =\int_{0}^{1 / 2} \bar{z}^{\prime} d s \\
& =z\left(\frac{1}{2}^{-}\right)-z(0) .
\end{aligned}
$$

Thus if $S=C$ applying Corollary 3.20 we find that one boundary condition is $z\left(\frac{1}{2}^{-}\right)=z(0)$. Since self-adjoint extensions $\sqrt{5}$ of $A$ satisfy the boundary condition $z(0)=z(1)$ we have also

$$
z(0)+z\left(\frac{1}{2}^{+}\right)-z\left(\frac{1}{2}^{-}\right)=z(1)
$$


so that $z\left(\frac{1+}{2}\right)=z(1)$. On the other hand if $S$ is trivial the boundary conditions are $z(1)=z(0)$ and $z\left(\frac{1}{2}+\right)-z\left(\frac{1}{2}^{-}\right)=0$.

This method is a general one and can be applied to more difficult examples which we will consider systematically elsewhere.

5. The adjoint of $\mathscr{A}_{I I}$. We end the paper with some remarks on the adjoint theory of (1.1) when $r \neq \theta$.

Definition 5.1. Let $\mathscr{A}_{H}: D(A) \rightarrow Y \times F$ and $\mathscr{A}_{H}^{+}: D_{H}^{+} \times F^{*} \rightarrow X^{*}$ be given respectively by $\mathscr{A}_{I I} y=(A y, H y)^{t}$ and $\mathscr{A}_{I I}^{+}(z, \phi)=A^{*} \bar{z}$ where $\phi \in \phi(z)$.

It is trivial that $\mathscr{A}_{H}$ and $\mathscr{A}_{H}^{+}$are densely defined operators.

Lemma 5.2. $\left[\mathscr{A}_{I I} y,(z, \phi)\right]=\left[y, \mathscr{A}_{H}^{+}(z, \phi)\right]$ on $D(A) \times D\left(A_{H}^{+}\right)$.

Proof. Immediate from Definition 5.1, Theorem 3.3, and the definition of an inner product on $(Y \times F) \times\left(Y^{*} \times F^{*}\right)$ (see $\S 2$ ).

The main result of this section is the following:

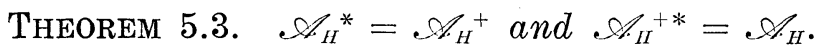

Proof. By Lemma $5.2 \mathscr{\mathscr { A }}_{I I}^{+} \subset \mathscr{\mathscr { A }}_{H}{ }^{*}$ and $\mathscr{A}_{H} \subset \mathscr{A}_{I}^{+*}$. Suppose $((\alpha, \dot{\phi}), \beta) \in G\left(\mathscr{C}_{H}{ }^{*}\right)$. Then

$$
[A y, \alpha]+[H y, \phi]=[y, \beta] .
$$

On the other hand $\phi \in \phi(z)$ for some $z$ in $D_{H}^{+}$. By Lemma 5.2

$$
[A y, z]+[H y, \dot{\phi}]=\left[y, A^{*} \bar{z}\right] .
$$

Subtracting (5.2) from (5.0) we find that

$$
[A y, \alpha-z]=\left[y, \beta-A^{*} \bar{z}\right] .
$$

Thus $\alpha-z \in D\left(A^{*}\right), \forall \alpha \in D_{H}^{+}$and

$$
\begin{aligned}
\beta & =A^{*} \bar{z}+A^{*}(\alpha-z) \\
& =A^{*}(\bar{\alpha}) .
\end{aligned}
$$

We conclude that $G\left(\mathscr{A}_{H}^{*}\right) \subset G\left(\mathscr{A}_{H}^{+}\right)$. Since $H$ is continuous on $G(A)$ when $F$ is endowed with the weak topology, $\mathscr{A}_{H}$ is easily verified to be closed. Hence $\mathscr{A}_{H}^{+*}=\mathscr{A}_{H}$.

REMARK 5.4. Note the adjoint theory for nonhomogeneous b.v.p. is much simpler than for $A_{H}$ in that $\mathscr{A}_{H}^{+}$are always closed operators and that there are no analogues of $\widetilde{A}_{I I}^{+}$. 


\section{REFERENCES}

1. R. Arens, Operational calculus of linear relations, Pacific J. Math., 11 (1961), 9-23.

2. R. C. Brown, The operator theory of generalized boundary value problems, Canad.,

J. Math., 28 (1976), 486-512.

3. — Differential operators with abstract boundary conditions, Canad. J. Math. 30 (1978), 262-288.

4. Caradus, Perturbation theory for generalized Fredholm operators, Pacific J. Math., 52 (1974), 11-15.

5. E. A. Coddington and A. Dijksma, Selfadjoint subspaces and eigenfunctions expansions for ordinary differential subspaces, J. Differential Equations, 20 (1976), $473-526$.

6. - Adjoint subspaces in Banach spaces. with applications to ordinary differential subspaces, to appear in Ann. di. Mat. Pura ed Appl.

7. N. Dunford and J. T. Schwartz, Linear Operators, Part I, Interscience, New York, 1957.

8. S. Goldberg, Unbounded Linear Operators: Theory and Applications, McGraw-Hill, New York, 1966.

9. S. J. Lee, Coordinatized adjoint subspaces in Hilbert spaces with applications to ordinary differential operators, to appear.

10. W. Rudin, Functional Analysis, McGraw-Hill, 1973.

Received July 31, 1978.

The University of Alabama

UNIVERSITY, AL 35486 


\section{PACIFIC JOURNAL OF MATHEMATICS}

\section{EDITORS}

DONALD BABBITT (Managing Editor)

University of California

Los Angeles, CA 90024

HUgo RossI

University of Utah

Salt Lake City, UT 84112

C. C. Moore and ANDrew OGG

University of California

Berkeley, CA 94720

\section{J. DUGUNDJI}

Department of Mathematics

University of Southern California

Los Angeles, CA 90007

R. FinN and J. MILGRaM

Stanford University

Stanford, CA 94305

\section{ASSOCIATE EDITORS}
E. F. BECKENBACH
B. H. NeumanN
F. WOLF
K. YOSHIDA

\section{SUPPORTING INSTITUTIONS}

UNIVERSITY OF BRITISH COLUMBIA CALIFORNIA INSTITUTE OF TECHNOLOGY

UNIVERSITY OF CALIFORNIA

MONTANA STATE UNIVERSITY

UNIVERSITY OF NEVADA, RENO

NEW MEXICO STATE UNIVERSITY

OREGON STATE UNIVERSITY

UNIVERSITY OF OREGON
UNIVERSITY OF SOUTHERN CALIFORNIA

STANFORD UNIVERSITY

UNIVERSITY OF HAWAII

UNIVERSITY OF TOKYO

UNIVERSITY OF UTAH

WASHINGTON STATE UNIVERSITY

UNIVERSITY OF WASHINGTON

The Supporting Institutions listed above contribute to the cost of publication of this Journal, but they are not owners or publishers and have no responsibility for its content or policies.

Mathematical papers intended for publication in the Pacific Journal of Mathematics should be in typed form or offset-reproduced, (not dittoed), double spaced with large margins. Please do not use built up fractions in the text of the manuscript. However, you may use them in the displayed equations. Underline Greek letters in red, German in green, and script in blue. The first paragraph or two must be capable of being used separately as a synopsis of the entire paper. Please propose a heading for the odd numbered pages of less than 35 characters. Manuscripts, in triplicate, may be sent to any one of the editors. Please classify according to the scheme of Math. Reviews, Index to Vol. 39. Supply name and address of author to whom proofs should be sent. All other communications should be addressed to the managing editor, or Elaine Barth, University of California, Los Angeles, California, 90024.

50 reprints to each author are provided free for each article, only if page charges have been substantially paid. Additional copies may be obtained at cost in multiples of 50 .

The Pacific Journal of Mathematics is issued monthly as of January 1966. Regular subscription rate: $\$ 84.00$ a year (6 Vols., 12 issues). Special rate: $\$ 42.00$ a year to individual members of supporting institutions.

Subscriptions, orders for numbers issued in the last three calendar years, and changes of address should be sent to Pacific Journal of Mathematics, P.O. Box 969, Carmel Valley, CA 93924, U.S.A. Older back numbers obtainable from Kraus Periodicals Co., Route 100, Millwood, NY 10546.

PUBLISHED BY PACIFIC JOURNAL OF MATHEMATICS, A NON-PROFIT CORPORATION

Printed at Kokusai Bunken Insatsusha (International Academic Printing Co., Ltd.). 8-8, 3-chome, Takadanobaba, Shinjuku-ku, Tokyo 160, Japan.

Copyright (C) 1979 by Pacific Journal of Mathematics Manufactured and first issued in Japan 


\section{Pacific Journal of Mathematics}

\section{Vol. 85, No. $2 \quad$ October, 1979}

Charles A. Akemann and Steve Wright, Compact and weakly compact derivations of $C^{*}$-algebras ........................... 253

Dwight Richard Bean, Andrzej Ehrenfeucht and George Frank McNulty, Avoidable patterns in strings of symbols ................... 261

Richard Clark Brown, Notes on generalized boundary value problems in Banach spaces. I. Adjoint and extension theory.................

Kenneth Alexander Brown and John William Lawrence, Injective hulls of group rings .................................... 323

Jacob Burbea, The Schwarzian derivative and the Poincaré metric ....... 345

Stefan Andrus Burr, On the completeness of sequences of perturbed polynomial values ....................................

Peter H. Chang, On the characterizations of the breakdown points of quasilinear wave equations..............................

Joseph Nicholas Fadyn, The projectivity of $\operatorname{Ext}(T, A)$ as a module over

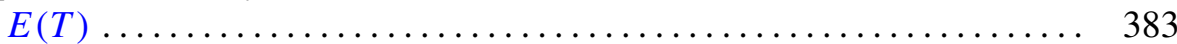

Donald Eugene Maurer, Arithmetic properties of the idèle discriminant .... 393

Stuart Rankin, Clive Reis and Gabriel Thierrin, Right subdirectly irreducible semigroups ................................. 403

David Lee Rector, Homotopy theory of rigid profinite spaces. I ........ 413 Raymond Moos Redheffer and Wolfgang V. Walter, Comparison theorems for parabolic functional inequalities................

H. M. (Hari Mohan) Srivastava, Some generalizations of Carlitz's theorem

James Alan Wood, Unbounded multipliers on commutative Banach algebras....

T. Yoshimoto, Vector-valued ergodic theorems for operators satisfying norm

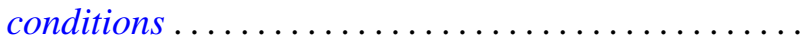

Jerry Searcy and B. Andreas Troesch, Correction to: "A cyclic inequality and a related eigenvalue problem".

Leslie Wilson, Corrections to: "Nonopenness of the set of Thom-Boardman maps" 